\title{
The significance of network literature in China and its value bearing System
}

\author{
Wu Binying ${ }^{1,}$, Wu Jinghui ${ }^{2, b}$ \\ ${ }^{1}$ Jiangxi Science \& Technology Normal University, Nanchang, Jiangxi, 330013, P.R. China \\ 2 East China Model High School, Shanghai, 200040, P.R. China \\ a ellen_wby@sina.com, ${ }^{b} 2658402638 @ q q . c o m$
}

Keywords: Network literature, Development core meaning, Value bearing system

\begin{abstract}
With the development of the times, the development of the society is obviously accelerated. Network literature has also been influenced, which is more unique, showing a more innovative development trend. At the same time, the network literature also presents some development problems, this paper makes an inquiry into the significance, value, problems and Strategies of the network literature.
\end{abstract}

\section{Introduction}

Under the current development situation, the development of Internet information technology, the network literature has become a popular art form of performance. With the accelerating social development rhythm, the network literature has been influenced by the network literature itself and not a clear boundary, and can not be divided into a strict class or bottom line, this kind of work is mainly based on the network as a medium, and formed a unique symbol of the writing features and characteristics, more consistent with the current situation of the network literature works. Network literature because of its specific development groups, hundreds of millions of Internet users have important significance.

\section{The value direction and significance of network literature}

From the macro, network literature as a cultural phenomenon, showing a certain period of time. For every person who loves reading, the network literature represents the meaning of certain times in different times hot. Today, China's network literature has formed a group of rising power.

With respect to the Japanese animation, South Korean television dramas, Chinese network literature showing a representation of the development of more Chinese, more Oriental. Since 2000, China's online literature works every year in the publication of the annual rate of increase of more than twenty-five percent. Now, into the country's major bookstores, are able to see the special set of network literature, a special counter. And in addition to the publication of paper books, the network literature is also more aspects of film and television drama, film, video games, animation and other different styles of change. The network literature is facing the popularization and the daily change. Comprehensive literature significance under the consumer market continue to drive, reflecting, for example, is more in line with the trend of the literary aesthetic, with strong color of realism, and the emergence of romance novels, novel of built on stilts, through the classification of the different novels, fantasy novels.

\section{Problems in the value of network literature}

Network literature to promote more people to achieve the dream of a civilian writer". Taiwan network writers Pizi Tsai's literary works "first intimate contact" let the works has become a symbol of the network literature development. And along with the development of network literature. Network romantic literature from the ruffian Cai here quickly became popular, from Taiwan to the mainland, the network literature became popular romance. The romantic color and rascal Cai works 
of different, different types of network romantic works, called network successful works number can be counted on one's fingers.

Because the network writers advocate free, relaxing, romantic, the network literature workers become a be the envy of the industry, more and more people to join in network writing. Some publishers in order to achieve greater economic benefits, began to implement the boundaries and scale of the network works, through the "civilian writers", so that the network literature works into a new development period.

The quality of the network is "uneven" . On the one hand, the network literature, "Chengdu, tonight, please forget me", "the other side" and other word of mouth works, on the other hand, it is also gradually exposed in addition to the mainstream, the challenges of the bottom line, the moral low, the value of the tilt, copy and imitate, and so many problems, seriously disrupted the spiritual value of literary writing. The fast food and pulp of literature thought is very serious. In many works, the literary structure is simple, the writing technique is naive and vulgar, and the development of the network literature is very loose, so this also leads to the creation of the network, the phenomenon is not enough. The development level of network literature and traditional literature still can not reach a certain depth.

The development of the categories of network literature "more and more mystery". With the hit "Zhen Huan biography" startling step by step "'" spend thousands of bone, such as the costume drama, the development of network literature are derived from the more branches. "And the day" and so on the day of the flight attendant cohabitation "and so on the modern urban literature also develops along with it. Each year, the network literature to film and television drama to change, production reached 10 million. Compared with the traditional literature, the network literature has a relatively low starting point, and the writing space is also more extensive. This also enables the network literature to write the author's comprehensive quality to have the difference, some vulgar literature work also very easy to circulate. With the development of Internet technology, network literature has become extremely popular. Some literature, Xian Xia fantasy literature hot up. These literary works overall values is confusion, such as network literature in the bad guys are how tempered "tells the story of the" bad guys "hero" wow gold "love and hate, easily bring to others in order to make ourselves strong and at the expense of all the negative effect.

\section{The specific value and direction of the development of network literature}

With the development of the times, the network literature also through continuous upgrading, such as a butterfly metamorphosis of high-quality literary works.

The network literature has enlarged the creation space of a writer. In the development of network literature, the traditional text literature has become a literary phenomenon, but the traditional network literature is confined to the individual creation, which is not able to integrate into the creative process in the process of its development. The traditional literary works that have been formed are not capable of creating literary texts. In the network literature, each reader can interact with other readers, and can integrate many people's creative writing.

For example in the readme ruffian CAI can be found, his literature experienced the two months and eight days of network creation, a total of 34 episodes of the series, so in each posted this article, netizens praise and encouragement to the rascal Cai then write down the courage. He said that in the "first intimate contact"

After birth is not to say that forever fixed, many readers when reading the re creation, there has been a lot of "writing" and "Rewriting" version, which also makes the network literature have greater creative space.

The formation of a unique network language". The audience of the network literature is mainly young Internet users, they have more time to contact with the network, and the communication and innovation are relatively strong, the network literature is influenced by many network readers. 
On the network language in network literature can be described as mixed, but objectively, these subversive, does not the mainstream network language also formed a special style. We can't say that this style is right or wrong, but it is still a very encouraging and a lot of people with a lot of people. The network language, network literature, network songs but also because of the particularity of the not to stick to one pattern widely welcomed by the people. Today, the pressure increases, many Internet users are looking for a free and release in the network. Such as network language, some of the commonly used words "pears Shandong" "god horse floating clouds" wood "' injury can not afford to "woman man" and so on, these words and some from the Pinyin input method, some from the language homonym, so it has characteristics of the network era.

The combination of traditional literature and Internet Literature. Quality of network literature gathering and publishing phenomenon to rewrite the only media, the creation of the network literature, Sina blog, the end of the world forum, Baidu post bar and other exchange platforms can be exchanged. In the process of writing can also be use related to animation, music patterns produced into e-books, electronic magazine, or language of traditional publishing, network literature will become black and white.

Let the network literature also become the traditional literature, the idea and the artistic style of the creators of the integration of the combination of a variety of artistic style, and laid a myriad of possible. And in Beijing, ending the "network literature 10 years inventory", "here boy", "Chengdu tonight, forget me" home "' carnal "' no "' the mask "and other ten outstanding works won the award for best network literature, and rally publishing volumes, sales once soared.

Network literature has led to greater economic benefits. Network literature writers is a new emergence of a group of new culture, these people from different social class and educational background, especially due to the different backgrounds and vulnerable to market economy hard become an instant success. Have many good writers in order to allow themselves to become the focus of media attention, and constantly exclusion, talk about public relations, between the fame and flounder. But there is no lack of good network literature writers, can really sink in the heart, the surly make a fuss, some of the good reputation of the network literature surfaced, regardless of the original literary works "start Chinese net" "below banyan", are also in the focus on creating a good literary products, baby Anne's "23", Ning "Wulin rumored", regardless of is in the change of drama of movie and TV or in published on won the good economic benefits, literature and economics, to achieve a win-win situation.

\section{Conclusion}

In summary, the network literature has a certain development space, also has a myriad of audience. However, the network literature to seek a better development and breakthrough, need to start from the different aspects, infinite zoom in the creation and development of space. Literature workers need to standardize the development of "green" development trend, and expand the development of the network literature.

\section{References}

[1] L.X. Xia, Tian Wei. Analysis of the elements of the network copyright infringement act. legal system and society, 08, 2010

[2] Y. Xiao. Copyright and digital library in the network environment. Beijing: Beijing Library Press, 2012

[3] F. Jameson, postmodernism and cultural theory. Xi'an: Shaanxi Normal University press, 1986:189-190. 